\title{
Perineural Tumor Invasion and Its Relation with the Lymphogenous Spread in Human and Experimental Carcinoma of Bile Duct. A Computer-aided 3-D Reconstruction Study
}

\author{
Masanori Suzuki*, $\uparrow$, Tohru Takahashi*, Kiyoaki \\ Ouchi† and Seiki Matsuno $\dagger$ \\ * Department of Pathology, Institute of Development, Aging \\ and Cancer, Tohoku University, Sendai 980 and †the First \\ Department of Surgery, Tohoku University School of \\ Medicine, Sendai 980
}

\begin{abstract}
Suzuki, M., Takahashi, T., Ouchi, K. and Matsuno, S. Perineural Tumor Invasion and Its Relation with the Lymphogenous Spread in Human and Experimental Carcinoma of Bile Duct. A Computer-aided 3-D Reconstruction Study. Tohoku J. Exp. Med., 1994, 172 (1), 17-28 — The pathogenesis of perineural tumor invasion was studied by computer aided 3-D reconstruction of bile duct wall from two patients submitted to surgery for hepatohilar carcinoma, in order to analyze how and via what route carcinoma reaches the perineural spaces. Rabbits with VX2 carcinoma implanted in the wall of the common bile duct were also examined. It was found that carcinomas growing along the perineural spaces had abundant connections with the tumors growing outside the nerve, especially those lurking in the lymphatics. In an additional analysis on the wall tissues of bile duct from 35 patients operated for carcinoma, the degree of invasion into perineural spaces proved to correlate with that into lymphatics much higher than with venular invasion. Thus it is likely that tumors reach distant nerves mainly via lymphatics, i.e., forming satellite lymphogenous foci around nerves and then, as a second step, breaking into the perineural spaces. —— perineural invasion; bile duct carcinoma; histopathology; 3-D reconstruction
\end{abstract}

The perineural tumor invasion was first described in the middle of the nineteenth century (Cruveilhier 1835; Neumann 1862), and its characteristic spreading pattern has been a matter of interest. Pathologists' attention was focussed among other aspects on the easy accessibility of tumors to the minute slits around peripheral nerve bundles, the so-called perineural spaces. Here the tumors appear to face little resistance in growing along the nerve axis and extending to distant sites. In carcinoma of the bile duct, which is the subject of the present investigation, perineural invasion is especially apt to occur (Fig. 1),

Received March 12, 1993; revision accepted for publication December 18, 1993.

Address for reprints : Masanori Suzuki, MD, The First Department of Surgery, Tohoku

University School of Medicine, 1-1 Seiryo-machi, Aoba-ku, Sendai 980, Japan. 


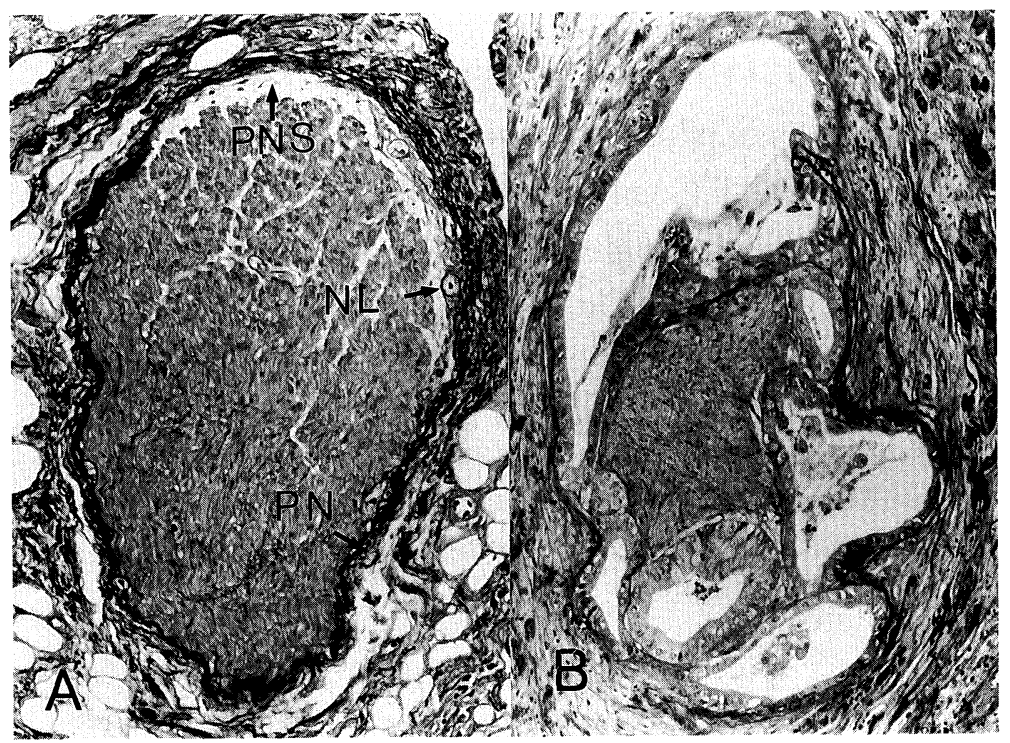

Fig. 1. Micrographs of the peripheral nerve and perineural tumor invasion. A: Microscopic structure of a peripheral nerve observed in the wall of bile duct (Azan-Mallory's stain, $\times 330$ ). A narrow slit, perineural space $(\mathrm{PNS})$, is formed between the nerve fibers and the perineurium (PN) of collagenous membrane. Specific inner lining of PN is called neurothelium (NL) or perineural epithelium. B: Perineural tumor invasion in carcinoma of bile duct (Elastica-Goldner's stain, $\times 200$ ). A peripheral nerve is completely surrounded by tubular adenocarcinoma.

and this explains why patients with this disease are so frequently tormented with refractory neuropathy. At the same time, the minute tumors that are dispersed far along the perineural spaces tend to escape eradication at radical surgery and remain as a potential source of recurrence. The susceptibility of the bile duct wall to neural involvement by carcinoma may be due to the affluence of peripheral nerves arising from the sympathetic trunk and the right branch of the vagal nerve; also responsible may be the anatomical thinness of the wall itself, which does not serve as a very firm barrier against tumors infiltrating from inside the duct. Information about the way tumors reach the perineural spaces may be of profound clinical importance. The route, if established in clear anatomical terms, would help design a strategy by which to deliver anticancer drugs effectively to minute tumors lurking in these spaces.

The question of whether or not perineural invasion is mediated via lymphatics or blood vessels, especially via the former, has long been discussed (Ernst 1905). Although the prevailing opinion today is that there is no continuity between perineural spaces and the neighboring lymphatics, ambiguities remain. In an effort to confirm the anatomical route along which one might send drugs most efficiently to perineurally infesting carcinoma cells, we have undertaken to 
visualize the pattern of extension of carcinoma in the wall tissue of bile ducts obtained at surgery, and also in those of rabbits with experimentally transplanted tumors. Since the basic task is to analyze the spatial relation between infiltrating tumors and perineural spaces, lymphatics and blood vessels of the bile duct wall, 3-D computer-aided reconstruction from serial sections was utilized to the extent possible. In addition, a study was performed from a clinicopathological viewpoint on what growth character of tumors correlates statistically with the degree of perineural invasion; this was done on surgical specimens from several patients with bile duct carcinoma.

\section{Materials ANd Methods}

The patients examined and the specimens

Surgical specimens for the reconstruction of infiltrating tumors were obtained from two patients undergoing surgery for hepatohilar bile duct carcinoma from whom a slice was taken by cross-sectioning the middle height of the common bile duct. In both of the patients, this portion was distant enough from the primary site so that, microscopically, the infiltrating nests of carcinoma proved to be sporadic, with the preformed wall structures including the nerves and lymphatics sufficiently intact. From the specimens, fixed in a $10 \%$ formalin solution and embedded in paraffin, approximately 1,000 serial histological sections $4 \mu \mathrm{m}$ thick were prepared. Most of the sections were stained with Elastica-Goldner stain, and the remainder with $\mathrm{HE}$ stain.

\section{Three- $D$ reconstruction}

In one of a set of serial sections, an area selected for reconstruction was projected onto a sheet of tracing paper at a magnification of $200 \times$ with a profile projector (model V-16C; Nikon, Tokyo), and the contours of the carcinoma nests, nerve bundles, lymphatics and blood vessels contained in the area were delineated as faithfully as possible. This was repeated for consecutive steps of serial sections so as to cover a thickness of $400 \mu \mathrm{m}$, which was attained by a consecutive treatment of 100 sections. Graphic reconstruction was performed with the aid of a computer system (PAS-310) specially designed for the study of 3-D microstructures at the Department of Pathology, Institute of Development, Aging and Cancer, Tohoku University. The system is based on a desktop computer (model 310, Hewlett-Packard, Fort Collins, CO., USA) with a color memory-mapped graphics display with $1024 \times 768$ pixels. The details of this system, including the hardware and the software, are to be referred to a previous article (Yaegashi et al. 1987). A drawing of 2-D profiles prepared as described above was placed on a digitizer where profiles of bile ducts, carcinoma cell nests, nerves, lymphatics and venules were inputted by tracing the contours with a cursor; this was repeated sequentially over the entire sequence of steps. The computer was then ready to integrate and display a 3-D picture rotated at any assigned angles around the $\mathrm{x}, \mathrm{y}$ and $\mathrm{z}$ axes, with the $\mathrm{xy}$ plane corresponding to the plane of section. Different structures such as nerve bundles, carcinoma cell nests and vessels were visualized with different colors. Two graphics techniques were combined in reproducing 3-D: the "stack of slices" and "wire framing." The former visualizes a structure as piled-up, painted discs with "hidden line" expression among them, while in the latter, only the contour lines were drawn, leaving the interior transparent. Shown in the following are 3-D pictures taken from the graphics display.

\section{Experimental study}

In addition, the growth behavior of tumors in the bile duct wall of rabbit was studied 
using experimentally transplanted VX2 tumor, a cell line estabished from a squamous cell carcinoma chemically induced in rabbit skin. A small volume (0.1-0.2 ml) of suspension containing cultured cells at a density of $10^{7}$ cells $/ \mathrm{ml}$ was injected into the subserosal tissue of the upper portion of the hepatoduodenal ligament of a male rabbit. The rabbit was killed on the seventh day, and a specimen was taken from the wall of the common bile duct, which was subjected in the same way as above to serial sectioning and 3-D reconstruction.

\section{Statistical analysis of factors involved in perineural invasion}

A clinico-pathological study was conducted on 35 patients with hepatohilar bile duct carcinoma submitted to surgery during the 22 years 1965-1986 at the 1st Department of Surgery, Tohoku University School of Medicine (Ouchi et al. 1989). On Elastica-Goldner stained microscopic sections of hilar bile ducts prepared from the surgical materials, the degree to which the tumors were growing in lymphatics (ly), venules (v) and perineural spaces (pn) were microscopically evaluated. The evaluation was made by assigning scores 0 to 3, conforming to the "General Rules for Cancer of the Biliary Tract" formulated by Japanese Society of Biliary Surgery (1993). To what degree the intensity of perineural invasion correlates with that of lymphatic or venular invasion was determined by Spearman's rank correlation test.

\section{Results}

\section{Perineurally growing carcinoma and its relation with the surrounding structures}

In performing 3-D reconstruction, attention was focussed on the anatomical relationship between the perineurally growing tumors and minute vessels in the

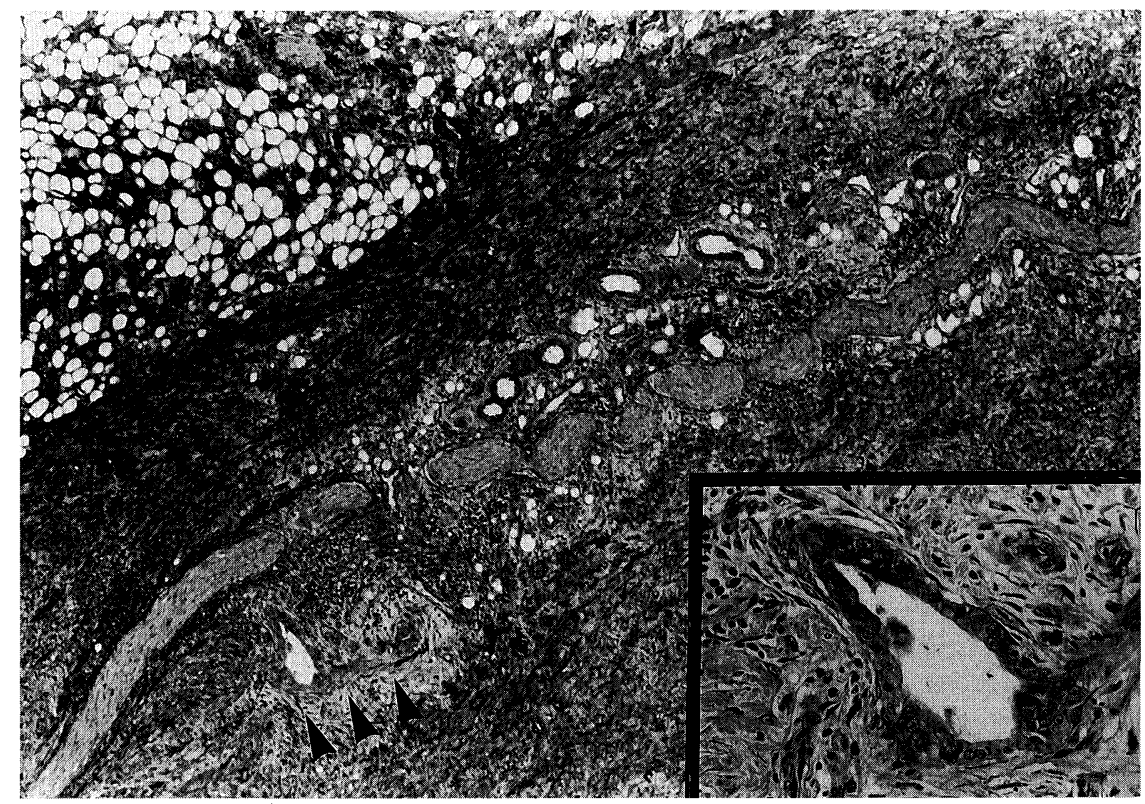

Fig. 2. Microscopic appearance of the wall of the common bile duct in Case 1, sample taken from a distant site from the primary tumor. A peripheral nerve is observed diagonally and shows invasion by carcinomatous nest in its left lower part (arrowheads). Inset (area in front of the arrowheads) shows carcinoma cells invading perineurally. (Elastica-Goldner's stain, $\times 200$ ). 
adjacent connective tissues. Fig. 2 is the microscopic appearance of the wall of the common bile duct in Case 1 at a site distant from the primary tumor. Nests of adenocarcinoma are found sporadically infiltrating around a bundle of peripheral nerve. This area was reconstructed in Fig. 6, a 3-D image taken from a computer display, where the draining vessels are seen forming a dense plexus around the nerve bundle. The "draining vessels" were so designated because the thin-walled small vessels around the nerve were hardly identifiable as venular or lymphatic segments. Apparently being continuous with the nerve bundle, these vessels seemed to drain the perineurium. In Fig. 3, another micrograph showing typical perineural invasion, a draining vessel is clearly demonstrated continuously leading to the perineural space where nests of carcinoma are harbored. This area is reproduced as a 3-D computer image in Fig. 7. Carcinomas (green) growing around the nerve bundles (yellow) are contiguous to and closely related with the draining vessels that form a dense network around the nerve. Fig. 4 is a micrograph from Case 2 showing a peripheral nerve in the bile duct wall, where nests of adenocarcinoma are growing in the small lymphatic spaces formed between the collagenous lamellae of the perineurium; in the surroundings, tumors are also found filling spaces that are mature lymphatic vessels in every respect, indicating that an advanced lymphatic invasion of carcinoma underlies perineural spread

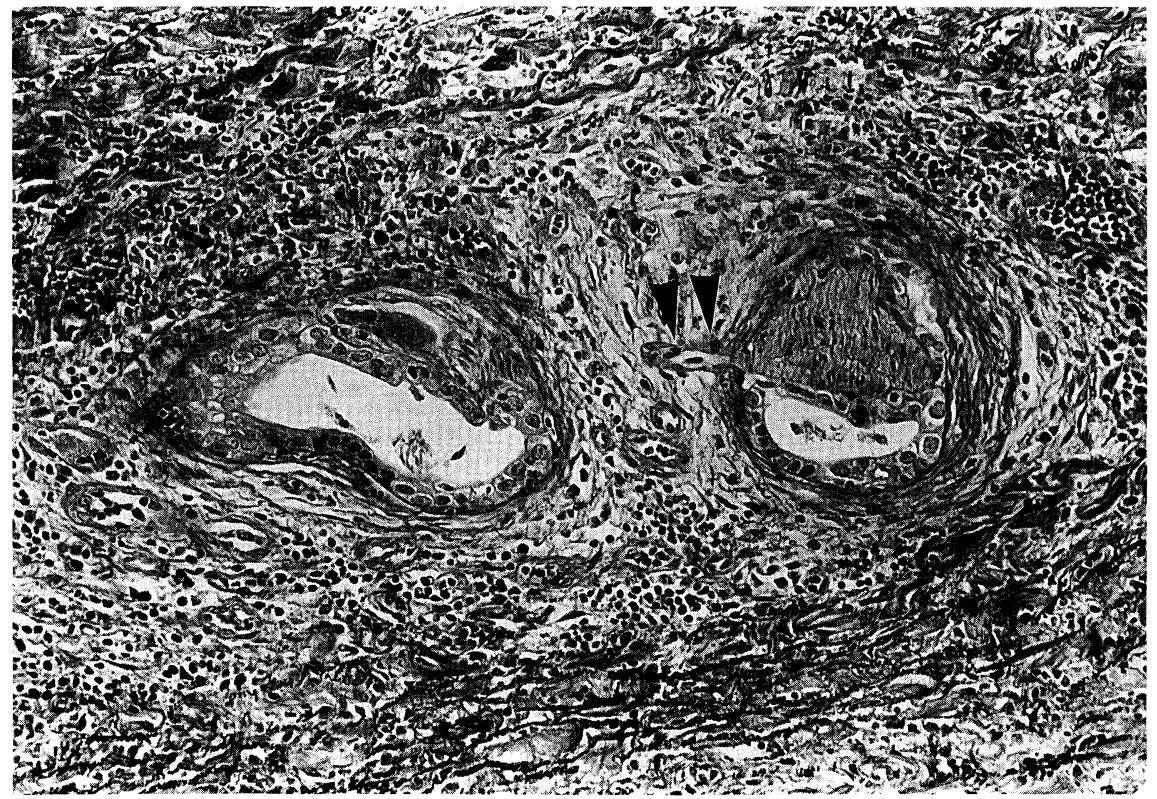

Fig. 3. Typical perineural invasion in Case 1. (Elastica-Goldner's stain, $\times 660$ ) Carcinomatous invasion with gland formation is observed inside the perineurium. A draining vessel is clearly demonstrated (arrowheads) continuously leading to the perineural space where nests of carcinoma are harbored. 


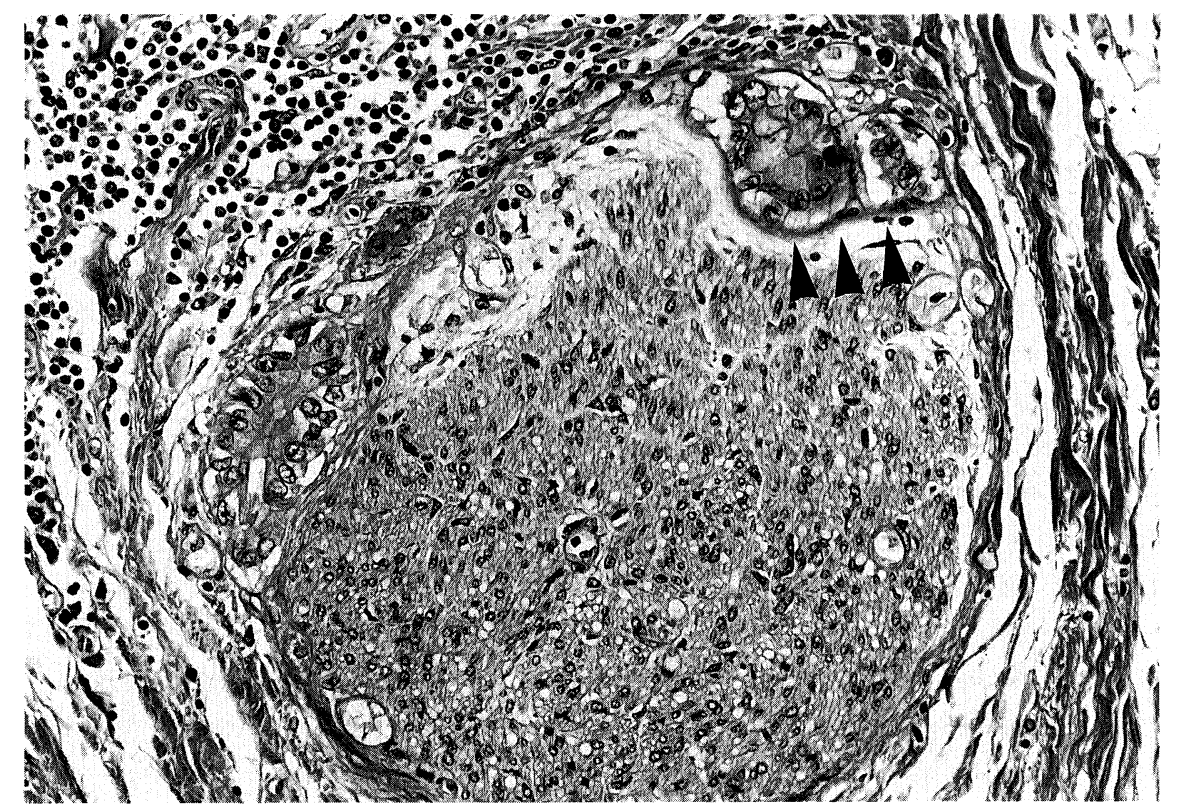

Fig. 4. Nests of adenocarcinoma captured within the small lymphatic vessel (arrowheads) between the collagenous lamellae of the perineurium in Case 2. Typical perineural tumor invasion is supposed to occur from this state. (HE stain $\times 660)$.

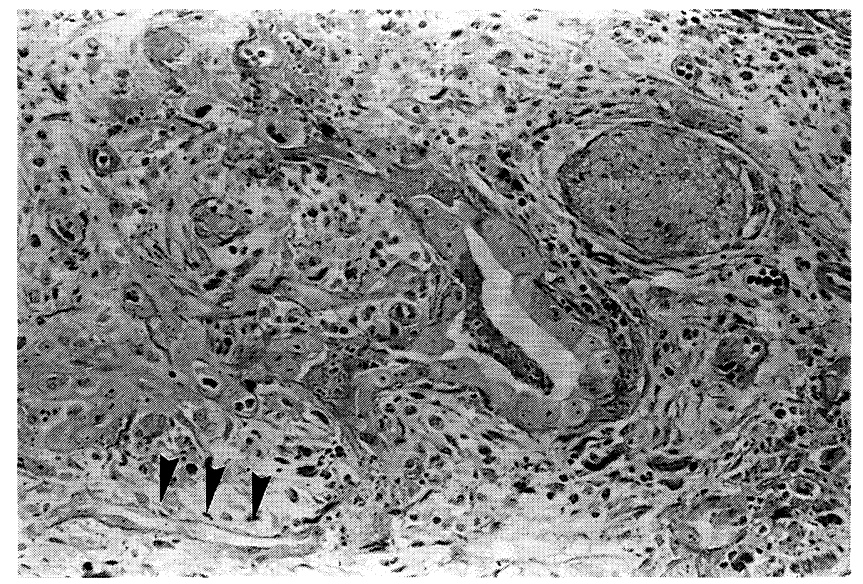

Fig. 5. Perineural invasion of carcinoma in Case 2. (Elastica-Goldner's stain, $\times 660$ ). Photomicrograph of the bile duct wall taken at a place far from the main tumor. Carcinoma growing out of the draining vessels (arrows) invades around the nerve bundle. 

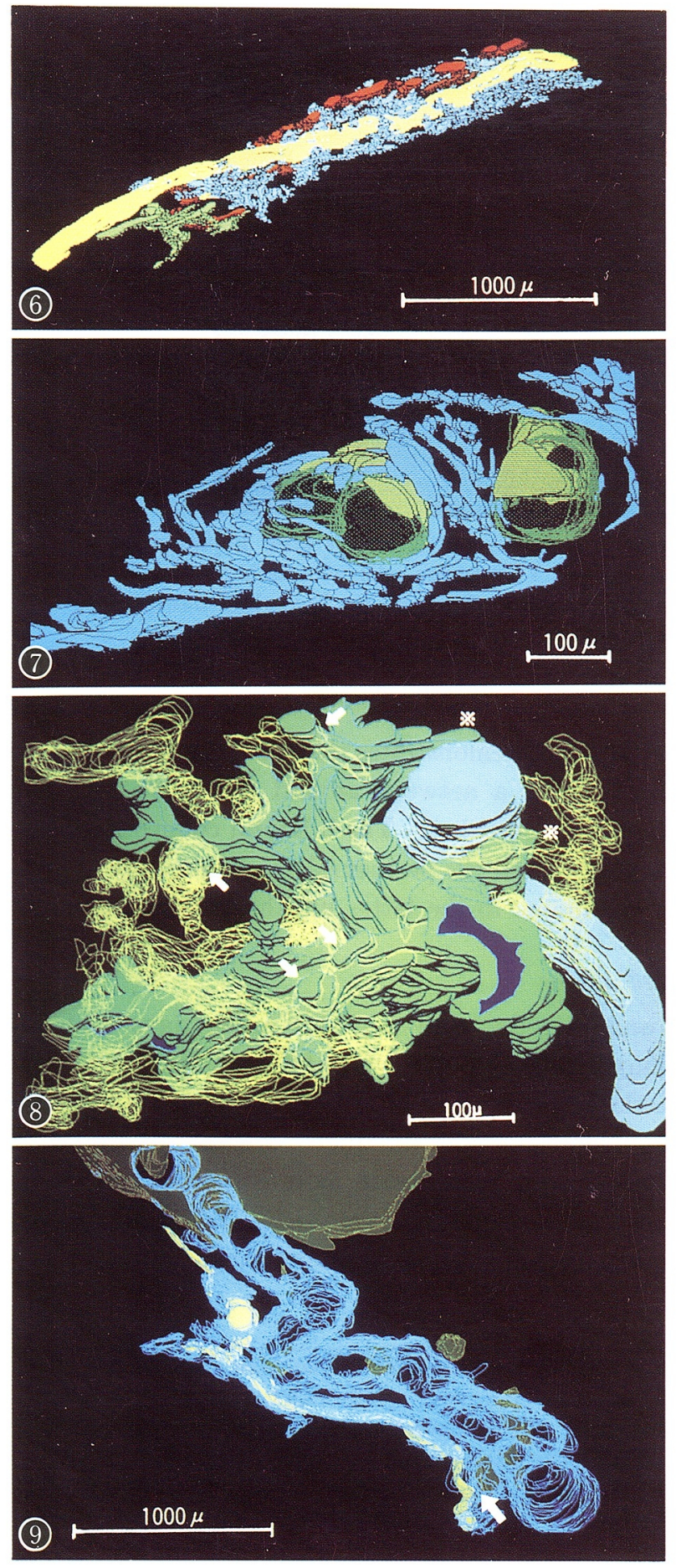

Fig. 6. Computer-aided reconstruction from the area of Fig. 2. The feeding arterioles (red) and the draining venules and lymphatics (light blue) are seen forming a dense plexus around the nerve bundle (yellow) with carcinomatous invasion (green).

Fig. 7. Computer-aided reconstruction of the perineurally growing carcinoma in the area of Fig. 3. Carcinoma (green) growing around the nerve bundles (yellow) are continuous to the draining vessels (light blue).

Fig. 8. Computer-aided reconstruction of the perineurally growing carcinoma in the area of Fig. 5. Carcinoma (green), growing out of the draining vessels (yellow wire-frames) at the points indicated by the arrows, forms a network aroud the nerve and then invades around the nerve (light blue) at the asterisks.

Fig. 9. Computer graphical 3D image from the area of Fig. 11C. Many small VX2 tumor cell clusters (green) are floating within the dilated lymphatic vessel (light blue wire-frame) and breaking its wall, invading the perineural space at the point indicated by the arrow (yellow). 


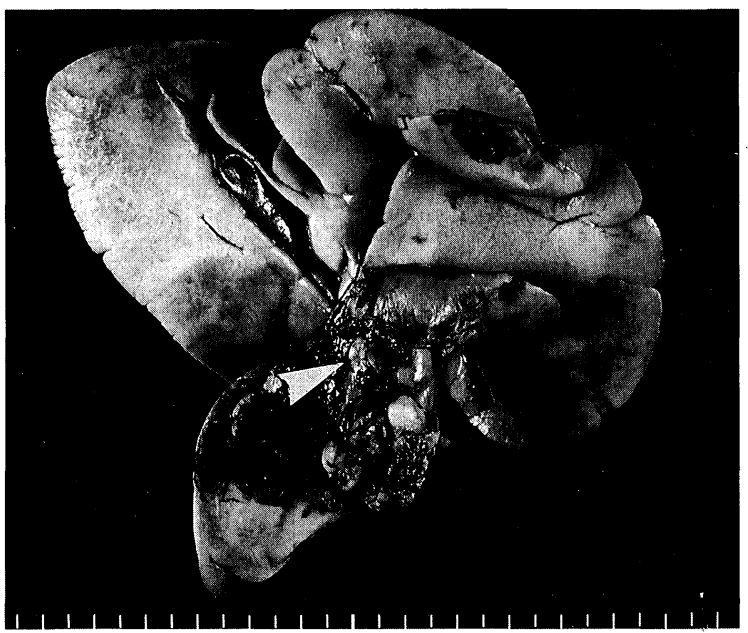

Fig. 10. Liver and bile ducts of a rabbit killed 7 days after implantation of suspension $0.1 \mathrm{ml}$ containing $10^{7} \mathrm{VX} 2$ tumor cells $/ \mathrm{ml}$ was injected into the subserosal tissue of the upper portion of bile duct. The white transplanted tumor is indicated by an arrow.

(Fig. 5). A 3-D reconstruction in Fig. 8 demonstrates that the tumor, growing out of the draining vessels (arrows), forms a network around the nerve and then penetrates into the perineural spaces (asterisks). The bilateral connection of the infiltrating tumor with the lymphatics and the perineural spaces strongly suggests an indirect but easy access of carcinoma to the latter via the lymphatics.

\section{The experimentally transplanted tumor}

Fig. 10 shows the liver and bile ducts of a rabbit seven days after the transplantation of VX2 carcinoma cells into the wall of the common bile duct. The tumor, forming a mass in the upper portion of the hepatoduodenal ligament, is spreading downward along the common bile duct. Microscopically, both lymphatic and perineural invasions were remarkable, as in Figs. 11A and B. In the surroundings of the tumor, as in Fig. 11C, lymphatic vessels around the peripheral nerves are considerably dilated. These are containing small clusters of

Fig. 11. Microscopic appearance of the transplanted hepatohilar carcinoma. A: Vascular invasion of VX2 carcinoma (HE stain, $\times 330$ ) with tumor cells proliferating in a dilated lymphatic vessel. B: Perineurally growing VX2 carcinoma ( $\mathrm{HE}$ stain, $\times 550$ ) with tumor cells proliferating around a peripheral nerve bundle. $\mathrm{C}$ : Histological appearance of the hepatoduodenal ligament neighboring the site of injected VX2 tumor. Lymphatic vessels surrounding the tumor are dilated, containing small clusters of tumor cells often destroying the walls of the lymphatics and infiltrating near the peripheral nerves (arrowheads). Inset shows an enlarged picture of the area pointed by the arrowheads. 

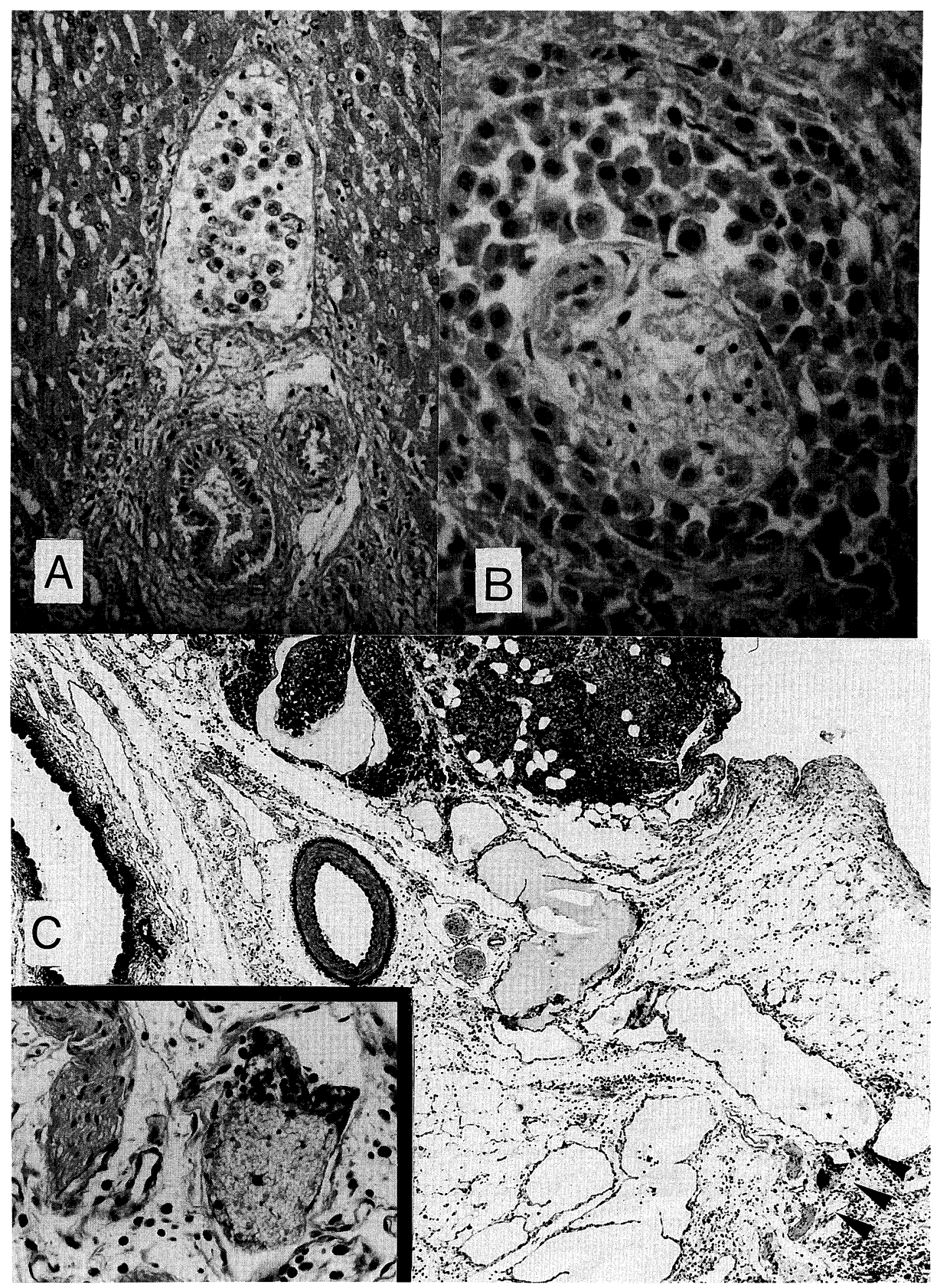

Fig. 11. 

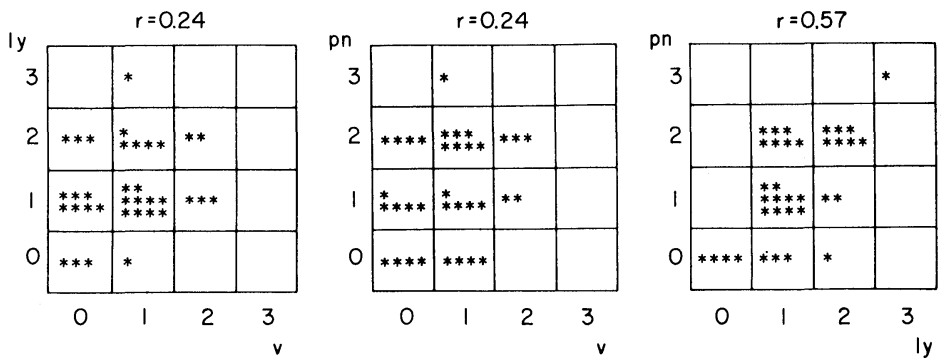

Fig. 12. Correlation between the grades of perineural and vascular invasion in surgical specimens of bile duct carcinoma. The degrees to which tumors are invading lymphatics (ly), venules (v) and perineural spaces (pn) were microscopically evaluated on 35 patients with hepatohilar bile duct carcinoma submitted to surgery. Correlations between v-ly, v-pn and ly-pn were examined by Spearman's method. The correlation coefficient between ly-pn proved to be higher than the others.

tumor cells which, often destroying the walls of the lymphatics, are infiltrating into the direct vicinity of a peripheral nerve (Fig. 11C). Fig. 9 is a $3-\mathrm{D}$ image showing the relation among the infiltrating tumors, lymphatics and a peripheral nerve. Lymphatic vessels are substantially engorged due to obstruction by the main tumor forming a nodule at the place of transplantation. In addition, tumor cell clusters are found attached to the wall of the lymphatic vessels, one of which is breaking the wall and entering the perineural space of the nerve. In this experimental model, perineural invasion by the tumor appears to have been mediated by its spread via the lymphatic vessels.

\section{Correlation between the grades of perineural and vascular invasion}

In surgical specimens of the common bile duct from 35 patients undergoing surgery for hepatohilar carcinoma, the intensity of carcinoma invasion into venules (v), lymphatics (ly) and perineural spaces (pn) was evaluated under microscope and classified among four grades ( 0 to 3 ), according to rules proposed by the Japanese Society of Biliary Surgery. Correlations between v-ly, v-pn and ly-pn were examined by Spearman's method (Fig. 12) with correlation coefficients calculated at $0.24,0.24$ and 0.57 , respectively, where the last alone is higher than the others. Although not statistically significant, the affinity of the lymphatics with the perineural spaces is particularly noticeable.

\section{Discussion}

In addition to the four major ways of spreading of abdominal cancers, namely focal invasion, lymphatic and hematogenous metastases and peritoneal dissemination, perineural invasion has often been referred to as the fifth important pathway. This implies extension of the carcinoma along the minute slit formed between the endoneurium and the perineurium, the latter being a thin collagenous membrane 
enclosing the bundle of the peripheral nerve. Although the study of perineural invasion dates back to Cruveilhier's first report published as early as 1835 (Ballantyne et al. 1963), this phenomenon was not a focus of pathologists' attention until 1905 when Ernst proposed to classify it among the major tumor spreading patterns (Ernst 1905). A series of papers followed, reporting a high frequency of perineural invasion in prostatic carcinoma, soft tissue sarcoma and carcinoma of the salivary gland (Ballantyne 1963; Stuart et al. 1971). Later, the highest frequency of perineural invasion was reported to be in the carcinomas of the pancreas and biliary tracts, organs equipped with an especially dense autonomic innervation (Miller 1951). Kuwayti et al. (1957), observing perineural invasion in as much as $61.7 \%$ of patients with pancreatic and bile duct carcinomas, regarded this as a highly specific feature of these tumors. The perineural spaces of the nerves were often called perineural lymphatics because they were considered to be continuous with and leading to the lymphatic system in the connective tissues surrounding the nerve. Larson et al. (1966), however, gave experimental findings inconsistent with this assumption. India ink injected into the lymphatics of animals was demonstrated not to reach the perineural spaces (Rodin et al. 1967). It has been assumed since then that the perineurium is an extension of the pia mater and pia arachnoidea of the spinal cord, acting as a barrier preventing circumferential micromolecules from diffusing into the nerve (Shanthaveerappa and Bourne 1966). Carcinoma, once entering this space, appears to face little resistance in growing longitudinally along the nerve axis, and this has been considered to account for the observation that in patients with bile duct carcinoma, tumor cells tend to reach unexpectedly remote place, even in early stages, where, too, the cells are found filling the perineural spaces. However, it is unlikely that carcinoma of the hilar bile duct progresses over a great distance such as that to the mesenteric and celiac nerve plexuses and occasionally, even around the aorta only by way of the perineural spaces. In this respect, it deserves special attention that in the present reconstruction, nerve bundles harboring carcinoma cells in their sheaths always proved to have a dense infiltration of tumor cells in the perineural connective tissues, too; frequently, these infiltrating carcinomas were shown to have connections with those growing in lymphatics on one hand, and on the other, with those in perineural spaces. This close association strongly suggests that the perineural invasion is a phenomenon assisted by simultaneous growth of carcinoma in the perineural connective tissues, especially in the lymphatics. Probably, carcinomas involving a nerve at a place far from their original site must have reached there via lymphatic vessels, forming a satellite focus around the nerve and then, as a second step, entered the nerve. Thus, one may say that the perineural invasion is a way of tumor spread mediated by lymphogenous extension. Support for this was also given by the result of experimental analysis using rabbit VX2 carcinoma cells. Although the cell stems from squamous cell carcinoma, its spreading behaviour via lymphogenous route is 
comparable to human bile duct carcinoma which usually is adenocarcinoma. Consequently, animal model using this tumor is useful in studying the mode of spread of bile duct carcinomas.

The result of the present investigation provides a key in designing a strategy for chemotherapy against hepatohilar bile duct carcinoma. It predicts a more favorable result of anticancer chemotherapy where drugs are delivered to perineurally growing carcinomas by way of lymphatics than by other means. Recently, Koyama et al. (1989) emphasize the effect of what they have established as a supplementary treatment for hepatohilar carcinoma, where, during surgical procedures, a suspension of active carbon-adsorbed mitomycin $\mathrm{C}$ is injected into the walls of the bile ducts. Via whatever route, drugs entering the lymphatics at a certain concentration may effectively work in eradicating carcinoma cells infesting the perineural spaces.

\section{References}

1) Ballantyne, A.J., Mc Cartten, A.B. \& Ibanez, M.L. (1963) The extention of cancer of the head and neck through peripheral nerves. Am. J. Surg., 106, 651-667.

2) Cruveilhier J. (1835) Maladies des nerfs. In: Anatomic Pathologique du Dorps Humain, 2nd ed., pt 35, p. 3. Paris, J.B. Bailliere.

3) Ernst, P. (1905) Über das Wachstum und die Verbreitung bösartiger Geschwülste, insbesondere des Krebses in den Lymphbahnen der Nerven. Beitr. Pathol. Anat., (suppl.) 7, 29-52.

4) Japanese Society of Biliary Surgery (1993) General Rules for Surgical and Pathological Studies on Cancer of Biliary Tract. 3rd ed., Kanehara publications, Tokyo.

5) Koyama, K., Tanaka, J., Kato, S. \& Asanuma, Y. (1989) A new strategy for treatment of carcinoma of the hilar bile duct. Surg. Gynecol. Obstet., 168, 523-530.

6) Kuwayti, K., Baggenstoss, A.H., Stauffer, M.H. \& Priestley, J.T. (1957) Carcinoma of the major intrahepatic and the extra-hepatic bile ducts. Exclusive of the papilla of Vater. Surg. Gynecol. Obstet., 104, 357-366.

7) Larson, D.L., Rodin, A.E., Roberts, D.K., O'Steen, W.K., Rapperport, A.S. \& Lewis, S.R. (1966) Perineural lymphatics - Myth or fact. Am. J. Surg., 112, 88-492.

8) Miller, J.R., Baggenstoss, A.H. \& Comfort, M.N. (1951) Carcinoma of the pancreas. Cancer, 4, 233-241.

9) Neumann, E. (1862) Secundäre Cancroid Infiltration des Nervus Mentalis bei einen Fall von Lippincroid. Arch. Pathol. Anat., 24, 201-202.

10) Ouchi, K., Suzuki, M., Hashimoto, L. \& Sato, T. (1989) Histological findings and prognostic factors in carcinoma of the upper bile duct. Am. J. Surg., 157, 552-556.

11) Rodin, A.E., Larson, D.L. \& Roberts, D.K. (1967) Nature of the perineural space invaded by prostatic carcinoma. Cancer, 2, 1772-1779.

12) Shanthaveerappa, J.R. \& Bourne, G.H. (1966) Perineural epithelium: A new concept of its role in the integrity of the peripheral nerve system. Science, 54, 1464-1467.

13) Stuart, W.L., Gaeta, J.F., Sako, K., Marchetta, F.C. \& Shedd, D.P. (1971) Adenocystic carcinoma of major and minor salivary glands. Am. J. Surg., 122, 756-762.

14) Yaegashi, H., Takahashi, T. \& Kawasaki, M. (1987) Microcomputer-aided reconstruction: A system designed for study of 3-D microstructure in histology and histopathology. J. Microsc., 145, 55-65. 